\title{
Efectos de una terapia basada en estimulación eléctrica funcional de activación bimanual combinada con el entrenamiento de biofeedback electromiográfico en la función motora de la extremidad superior parética en sujetos secuelados de accidente cerebrovascular.
} Effects of a therapy based on a functional electrical stimulation of bimanual activation combined with the training of electromyographic biofeedback on the motor function of the upper limb in subjects with stroke.

Sebastián Alarcón A. ${ }^{1 a}$, Barbara Sánchez O. ${ }^{1 a}$, Hachi Manzur V b , Julio Torres E 1a

\begin{abstract}
Introduction : Stroke is the leading cause of death and disability in Chile. More than $85 \%$ of patients suffer from hemiplegia and more than $69 \%$ functional motor disability of the upper extremities. This alteration generates an impact on the use of the upper limb, affecting the various activities of people's daily lives. There are no studies that combine electromyographic biofeedback therapy and bimanual activation with functional electrical stimulation in subjects with stroke. Therefore, there is interest in determining the effect of a training protocol based on Functional Electrical Stimulation (FES) with bimanual activation and biofeedback therapy on the function of the upper limb. Methodology: 15 subjects with stroke between 40 and 85 years recruited in the outpatients neurorehabilitation program of Clinica Dávila, randomized in an experimental group and in two control groups of 5 subjects. In each session the experimental group will train fifteen minutes of bimanual activation with functional electrostimulation and then a ten-minute biofeedback training program, while the control 1 and control 2 group trained under the same conditions but with placebo FES and placebo BF-EMG respectively. Results There were significant changes in the experimental group after the intervention. Conclusion This study suggests the electrical stimulation works and biofeedback as a tool for the rehabilitation of the upper limb in subjects with stroke.
\end{abstract}

Key words: Stroke, Neurofeedback, Rehabilitation

Rev Chil Neuro-Psiquiat 2020; 58 (2): 150-160

Aceptado: $14 / 04 / 2020$

Recibido: 04/12/2019

Kinesiólogo

Servicio de Medicina Física y Rehabilitación, Clínica Dávila.

Hospital Clínico Universidad Católica 


\section{Introducción}

$\mathrm{N}$ uestro país ha experimentado un gran cambio en su composición etárea en los últimos años, posicionándose como uno de los países más envejecidos de latinoamérica. Esto a su vez, ha traído modificaciones en la situación de salud de los Chilenos, donde el accidente cerebrovascular (ACV) es la principal causa de muerte, con 9.004 fallecidos el año 2013, lo que corresponde a una persona por hora. Se calcula que anualmente hay 24.964 casos nuevos (69 casos cada día) $)^{(1)}$.

Más del 85\% de los pacientes con ACV sufren hemiplejia, y más del $69 \%$ de ellos experimentan una discapacidad motora funcional de las extremidades superiores (2). Además, es la primera causa específica de años de vida saludables perdidos por discapacidad en mayores de 74 años. Por todo lo anterior, el ACV se constituye como un problema crítico de Salud Pública ${ }^{(1)}$.

Según la última guía clínica AUGE de Accidente cerebrovascular isquémico, en la población Chilena un $14 \%$ de los pacientes con paresia severa, con poco o sin movimiento activo al ingreso, experimentó una completa recuperación, mientras que un 30\% alcanzó una recuperación parcial. Este déficit genera impacto en el uso de la extremidad superior para la realización de alcances, prensión, manipulación, exploración ambiental, balance, afectando por tanto las diversas actividades de la vida diaria $^{(1)}$. Pese a todas las intervenciones, la discapacidad motora funcional aparece con frecuencia en las extremidades superiores en lugar de en las extremidades inferiores. Una de las razones puede ser la alta frecuencia con que la arteria cerebral media (ACM) o sus derivadas son afectadas en el ACV, cuyo territorio abarca áreas corticales correspondientes a las funciones motoras de las extremidades superiores $(75 \%$ de la extensión de territorio afectado por el ACV).
Lang et al señalaron que la discapacidad motora de las extremidades superiores afecta significativamente el rendimiento de los pacientes con ACV de las actividades de la vida diaria, como comer, usar ropa o lavarse la cara $^{(2,3)}$. Estas funciones de las extremidades superiores se han enfatizado como un elemento importante en los seres humanos (4). Desde esta perspectiva, esta enfermedad es un generador de importantes y diversas secuelas tanto del ámbito motor como cognitivo, que interfieren significativamente la calidad de vida de quienes la presentan. Existen variadas intervenciones terapéuticas para la recuperación motora de la extremidad superior, que van desde técnicas de neurodesarrollo, entrenamientos bimanuales, terapia de restricción del movimiento, entrenamientos de fuerza, terapia en espejo, práctica mental, biofeedback, tecnología de realidad virtual y terapia de movimiento asistido por dispositivos robóticos (Tabla 1) (1).

Se han realizado múltiples estudios previos utilizando distintas técnicas en forma combinada. Hyun Seok ${ }^{(3)}$ comparó dos grupos de sujetos secuelados de accidente cerebrovascular subagudo. El grupo experimental recibió terapia restrictiva combinada con biofeedback visual. Este último consistía en un dispositivo que proyectaba en la pantalla diferentes juegos conectado a un dinamómetro y un medidor de pellizco, sin utilizar EMG. El grupo control solo recibió terapia de biofeedback visual. No se encontraron diferencias significativas entre ambos grupos en todas las variables evaluadas; Test de Purdue Pegboard, prueba de fuerza de agarre JAMAR, Test de Wolf para función motora, EFM-ES,índice de motricidad, versión coreana del índice de Barthel modificado. Hasta la fecha no existen estudios que combinen terapia de biofeedback electromiográfico con EEF en sujetos secuelados de accidente cerebrovascular subagudo. 
Tabla 1. Análisis de resultados intergrupal

\begin{tabular}{|c|c|c|c|c|c|c|}
\hline & Exp & $\min -\max$ & Control 1 & $\min -\max$ & Control 2 & $\min -\max$ \\
\hline \multicolumn{7}{|l|}{ Fugl-Meyer } \\
\hline Antes & 32 & $14-58$ & 64 & $55-66$ & 60 & $40-63$ \\
\hline Después & 51 & $32-66$ & 64 & $12-66$ & 65 & $51-66$ \\
\hline Diferencia & 13 & $8-27$ & & -17 & 6 & -12 \\
\hline$P$ value & $*(p=0,04)$ & & $(p=0,7)$ & & $(p=0,078)$ & \\
\hline \multicolumn{7}{|l|}{ Sensibilidad } \\
\hline Antes & 7 & $10-12$ & 9 & $3-4$ & 10 & $8-12$ \\
\hline Después & 10 & $9-12$ & 12 & $10-12$ & 12 & $11-12$ \\
\hline Diferencia & 2 & $0-6$ & 3 & $2-6$ & 2 & $0-3$ \\
\hline$P$ value & $(p=0,1)$ & & $*(p=0,04)$ & & $(p=0,06)$ & \\
\hline \multicolumn{7}{|l|}{ Rango } \\
\hline Antes & 15 & $15-24$ & 24 & $21-24$ & 24 & $21-24$ \\
\hline Después & 24 & $15-24$ & 24 & $24-24$ & 24 & $21-24$ \\
\hline Diferencia & 7 & $0-9$ & 0 & $0-3$ & 0 & -6 \\
\hline P-value & $(p=0,1)$ & & $(p=0,3)$ & & $(p=1)$ & \\
\hline \multicolumn{7}{|l|}{ Dolor } \\
\hline Antes & 13 & $10-23$ & 19 & $15-23$ & 19 & $15-23$ \\
\hline Después & 20 & $15-24$ & 23 & $20-24$ & 24 & $20-24$ \\
\hline Diferencia & 3 & $1-13$ & 4 & -12 & 5 & $0-6$ \\
\hline$P$ value & $*(p=0,043)$ & & $(p=0,138)$ & & $*(p=0,066)$ & \\
\hline \multicolumn{7}{|l|}{ TMM FM } \\
\hline Antes & 3 & $3-5$ & 4 & $3-5$ & 3 & $3-4$ \\
\hline Después & 4 & $3-5$ & 5 & $4-5$ & 5 & $4-5$ \\
\hline Diferencia & 1 & $0-1$ & 0 & $0-2$ & 1 & $1-2$ \\
\hline$P$ value & $*(p=0,046)$ & & $(p=0,180)$ & & $*(p=0,034)$ & \\
\hline \multicolumn{7}{|l|}{ TMM EM } \\
\hline Antes & 3 & $3-4$ & 4 & $3-4$ & 3 & $3-4$ \\
\hline Después & 4 & $4-5$ & 5 & $4-5$ & 5 & $4-5$ \\
\hline Diferencia & 1 & $0-1$ & 1 & $0-2$ & 1 & $1-2$ \\
\hline$P$ value & $*(p=0,046)$ & & $(p=0,102)$ & & $*(p=0,034)$ & \\
\hline \multicolumn{7}{|l|}{ Ash FM } \\
\hline Antes & 0 & $0-1$ & 0 & $0-1$ & 0 & $0-1$ \\
\hline Después & 0 & $0-0$ & 0 & $0-0$ & 0 & $0-0$ \\
\hline Diferencia & 0 & & 0 & & 0 & \\
\hline $\mathrm{P}$ value & $(p=0,157)$ & & $(p=0,317)$ & & $(p=0,317)$ & \\
\hline \multicolumn{7}{|l|}{$<$ Ash EM } \\
\hline Antes & 0 & $0-1$ & 0 & $0-1$ & 0 & $0-1$ \\
\hline Después & 0 & $0-0$ & 0 & $0-0$ & 0 & $0-0$ \\
\hline Diferencia & 0 & & 0 & & 0 & \\
\hline$P$ value & $(p=0,317)$ & & $(p=0,317)$ & & $(p=0,317)$ & \\
\hline
\end{tabular}




\section{Biofeedback}

En esta técnica, las señales biológicas son presentadas al usuario mediante un estímulo visual. Se recomienda el uso de bio retroalimentación visual para mejorar funciones y rendimiento motor de la extremidad superior en secuelados de accidente cerebrovascular ${ }^{(5,6,7)}$, pero aún la dosis de biofeedback no ha sido un parámetro investigado ${ }^{(8)}$. En un metaanálisis, Moreland $y$ Thomson encontraron un incremento pequeño en la funcionalidad de extremidad superior usando biofeedback en comparación con la terapia convencional ${ }^{(8)}$.

Para este estudio se utilizó el dispositivo EMGOne, que es un dispositivo chileno basado en biofeedback electromiográfico (BF-EMG), de bajo costo, que permite la detección de la actividad muscular, la amplifica y la exhibe a modo de retroalimentación mediante un videojuego visualizado en una tablet. Esto favorece la propiocepción $^{(9)}$ y la motivación ${ }^{(7)}$, mejora el aprendizaje motor, la atención ${ }^{(5)}$ y funciones tanto del miembro superior ${ }^{(1,3,8)}$ como inferior ${ }^{(10,11,12)}$ con resultados más efectivos en menor tiempo ${ }^{(13,14,15)}$, aunque es posible encontrar estrategias compensatorias de movimiento ${ }^{(17)}$.

Electroestimulación funcional (EEF): Es una técnica de estimulación muscular por medio de corriente eléctrica aplicada por un sistema de electrodos superficiales que inducen una contracción muscular inmediata de los músculos esqueléticos para lograr movimientos funcionales ${ }^{(18)}$. Aumenta el movimiento activo después del accidente cerebrovascular y tiene mejor evidencia en la etapa subaguda ${ }^{(19.20,21)}$. Reduce la espasticidad y mejora activación, rango y funcionalidad en una muñeca hemiparética ${ }^{(22)}$ Sin embargo, faltan mayores investigaciones para llegar a resultados concluyentes. ${ }^{(23)}$. Para este estudio se utilizó TrainFes, un dispositivo de EEF creado por la compañía chilena TrainFes ${ }^{\circledR}$, que proporciona distintas modalidades de tratamiento. En este estudio se realizará un registro posicional de la extremidad sana que al detectar el movimiento de extensión de muñeca, gatillara el mismo movimiento en la extremidad parética mediante la estimulación directa de los músculos involucrados.La forma de onda es bifásica simétrica cuadrada, con control por corriente y compensada.. La intensidad va de $0-130 \mathrm{~mA}$, con pasos de 1mA, una frecuencia: $1-60 \mathrm{~Hz}$ y un ancho de pulso: $20-400 u S$ por fase. Todos estas especificaciones garantizan seguridad para los tejidos con ondas compensadas, que implica que la sumatoria de cargas (Q) aplicadas es 0 , por lo que debe tener 2 polaridades, y la sumatoria de las cargas de la fase positiva y las de la negativa deben ser iguales para que se anulen. Esto, permite que no haya acumulación neta de carga en los tejidos, de no ser así, las cargas netas acumuladas generan efectos químicos sobre los tejidos. Además, TrainFES ${ }^{\circledR}$ cuenta con estándares internacionales, cumpliendo con norma ISO13485 e IEC606001.

La electroestimulación funcional está siendo muy aplicada con buena evidencia, pero consideramos que se puede complementar con otro tipo de terapias. Es importante poder hacer este estudio ya que actualmente no existen estudios que combinen terapia convencional, estimulación eléctrica funcional $y$ biofeedback electromiográfico y en sujetos secuelados de accidente cerebrovascular. Sumado a esto, y al impacto que tiene la función de la extremidad superior en la calidad de vida de los sujetos, es que surge el interés de determinar el efecto que tiene un protocolo de entrenamiento basado en terapia convencional, estimulación eléctrica funcional (EEF) con activación bimanual y terapia de biofeedback en la función de la extremidad superior. 


\section{Hipótesis}

La asociación del protocolo de entrenamiento a la terapia convencional, estimulación eléctrica funcional y biofeedback electromiográfico produce resultados favorables en la activación y la función de apertura de la mano parética en los sujetos secuelados de accidente cerebrovascular subagudo.

\section{Objetivo}

Evidenciar la eficacia de un protocolo de intervención de terapia convencional, electroestimulación funcional y biofeedback en sujetos secuelados de accidente cerebrovascular subagudo en la funcionalidad de la extremidad superior parética.

\section{Metodología}

\section{Diseño}

Los procedimientos de esta investigación cuantitativa, longitudinal, de tipo experimental controlado randomizado. respetaron las normas éticas concordantes con la Declaración de Helsinki (1975), actualizadas en el 2008. Este estudio y el consentimiento informado del mismo, fueron aprobados por el comité de ética del servicio metropolitano de salud norte y de Clínica Dávila, lugar en el cual se llevó a cabo a la investigación.

\section{Muestra}

Según estudios que utilizaron biofeedback y estimulación eléctrica funcional, se necesitan mínimo 30 sujetos $^{(2)(3)}$. Considerando que se trata de un estudio piloto, se midieron 15 sujetos entre Marzo a Octubre del 2019.

Criterios de inclusión; Sujetos cooperadores edad entre 40 y 85 años secuelados de ACV isquémico supratentorial subagudo, entre 7 días a 6 meses de evolución, con secuela de hemiparesia de la extremidad superior, con activación M3 en el test manual muscular para todos los grupos musculares de la mano, con
0 y 1+ en la escala de Ashworth modificada $\mathrm{y}$ que mantengan posición sedente. Criterios de exclusión fueron; Alteraciones visuales,sensoriomotora de la extremidad superior indemne, accidente cerebrovascular previo, Alteraciones músculo-esqueléticas, cirugías o impotencia funcional por dolor EVA $8 / 10$ que no permitan movilizar la mano parética.

\section{Randomización}

La aleatorización fue mediante una pecera en tres grupos: Experimental, Control $1 \mathrm{y}$ Control 2, en el cual finalmente para cada grupo se asignaron 5 sujetos. (Figura 1)

\section{Cegamiento}

Este estudio es doble ciego. El evaluador fue ciego al inicio, al terminar la segunda, la cuarta semana y luego de un mes de seguimiento será ciego para las siguientes mediciones en la extremidad superior más afectada; test muscular manual (TMM) junto a la escala de Ashworth modificada para los flexo extensores de muñeca, y la escala de Fugl-Meyer ${ }^{(25)}$ de extremidad superior (EFM-ES). Los sujetos también fueron ciegos. El análisis de datos también lo realizó un estadístico ciego al finalizar la terapia y al finalizar el seguimiento. Solo el terapeuta que aplicaba la terapia no era ciego.

\section{Intervención}

Tuvo una duración de cuatro semanas, tres veces por semana. Cada sujeto estaba sentado en una silla con respaldo, con una mesa por anterior y los brazos en posición de quince grados de hombro, noventa grados de flexión de codo y cero grados de muñeca. En cada sesión el grupo experimental entrenó en primer lugar 30 minutos de terapia convencional que consistió en el $75 \%$ del tiempo en activación neuromusculares,dos ejercicios por grupo muscular, en primer lugar sin resistencia y segundo lugar una 
Figura 1. Flujograma de la investigación

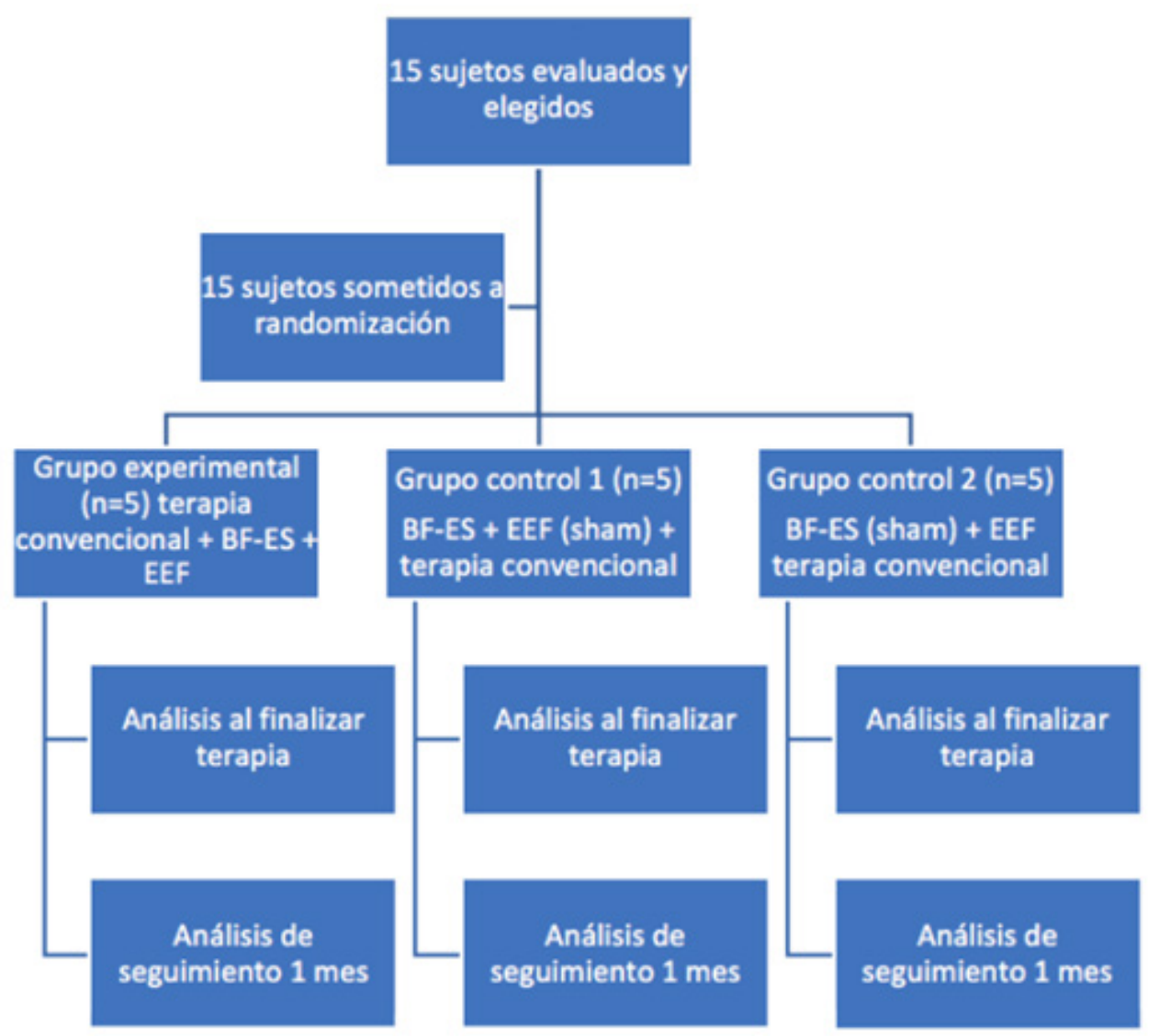

resistencia moderada. Por cada ejercicio fueron cincuenta repeticiones. Los grupos musculares a entrenar fueron: flexoextensión de muñeca y dedos y de pronosupinación. El otro $20 \%$ de la terapia fue de flexibilizaciones sostenidas de veinte segundos por cada grupo muscular; flexores, extensores, pronadores, supinadores. Finalmente el 5\% de la terapia, en la práctica se realizó estimulación sensitiva protopática y epicrítica con cuatro elementos; un cepillo, una pelota con puntas plásticas, una esponja y un vibrador por todo el antebrazo, muñeca y dedos, con una dosificación de 20 repeticiones por cada elemento.

Luego, entrenaron quince minutos de activación bimanual con electroestimulación funcional, donde cada sujeto realizó veinte repeticiones de extensión de muñeca bimanual dentro de un minuto, sujetando dos conos con una argolla en el medio la cual debieron mantener en dicha posición durante todo el movimiento extensor y con descansos de un minuto en posición de reposo sin realizar activación. Finalmente, se sometieron a un programa de entrenamiento de biofeedback EMGONE 10 minutos, el cual consistió en sortear obstáculos que se encontraban en la parte inferior de la pantalla tablet a una velocidad de 0.5 en la aplicación, gravedad normal de $1.0 \mathrm{x}$ en la configuración, con obstáculos que aparecen cada cinco segundos, y con un tamaño de 50 $\mathrm{cm}$.

En cuanto a los grupos controles, el grupo control 1 entrenó en las mismas condiciones 
pero la electroestimulación funcional fue placebo con una intensidad subóptima mientras que el grupo control 2 también recibió el mismo tratamiento que el grupo experimental pero la administración de biofeedback electromiográfico placebo con una calibración previa submaximal. (Figura 2).

Figura 2. Terapia basada en uso de electroestimulador funcional bimanual con biofeedback electromiográfico.

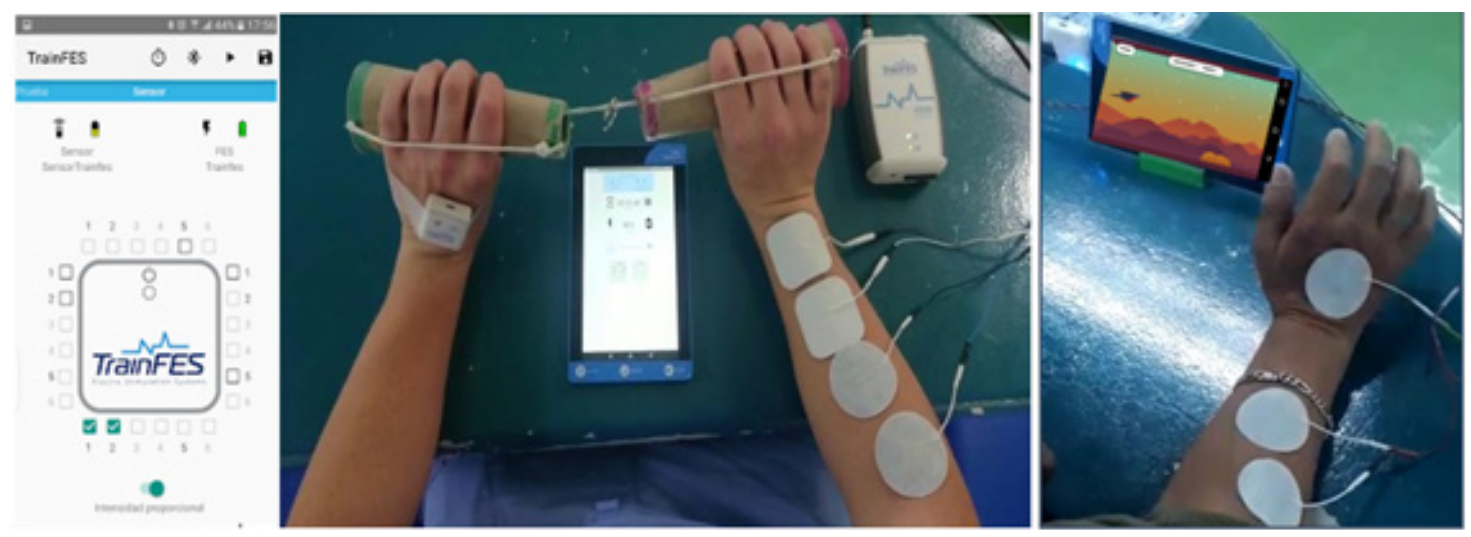

\section{Resultados}

\section{Estadística}

Se utilizó el test de Willcoxon para comparar los resultados entre los grupos y los valores antes y después del tratamiento para todos los sujetos. Los resultados se expresaron en medianas con el valor mínimo y máximo. Fue considerado el nivel de significancia estadística $\mathrm{p}<0,05$ para la comparación de las variables pre y post tratamiento y entre los tres grupos de estudio. (Tabla1).

\section{Fugl-Meyer test}

En el grupo experimental, hay una diferencia en mediana de 13 puntos, y en los los dos grupos controles que no existieron cambios significativos para este test.

En total, once sujetos aumentaron su puntaje en la escala; todos los sujetos del grupo experimental $(100 \%)$, cuatro sujetos del grupo control $2(80 \%)$ y solo uno del grupo control 1 (20\%). (Figura 3).

Solo existieron dos outliers, uno para el grupo control $1 \mathrm{y}$ otro para el grupo control 2.
$T M M$

Para el grupo de extensores de muñeca solo los cambios estadísticamente significativos fueron para el grupo experimental y el grupo control 2. En cuanto al grupo control 1 no existieron cambios significativos. Este mismo fenómeno ocurrió para los grupos de flexores de muñeca (Figura 4). Solo existieron dos outliers, uno para el grupo experimental y otro para el grupo control 1.

\section{Ashworth}

No existieron cambios significativos para el tono en ninguno de los grupos musculares. Todos los sujetos finalizaron el protocolo de investigación con Ashworth 0 en todos los grupos musculares.

\section{Conclusión}

La combinación de las tres intervenciones sugiere tener un mayor impacto en la función de la mano de los sujetos secuelados de accidente cerebrovascular subagudo.

Aun así, no existieron cambios en la sensibilidad, por lo que solo la sugerencia 
Figura 3. Comparación de los cambios para Fugl-Meyer test de la extremidad superior parética en los diferentes grupo antes y después de la intervención.

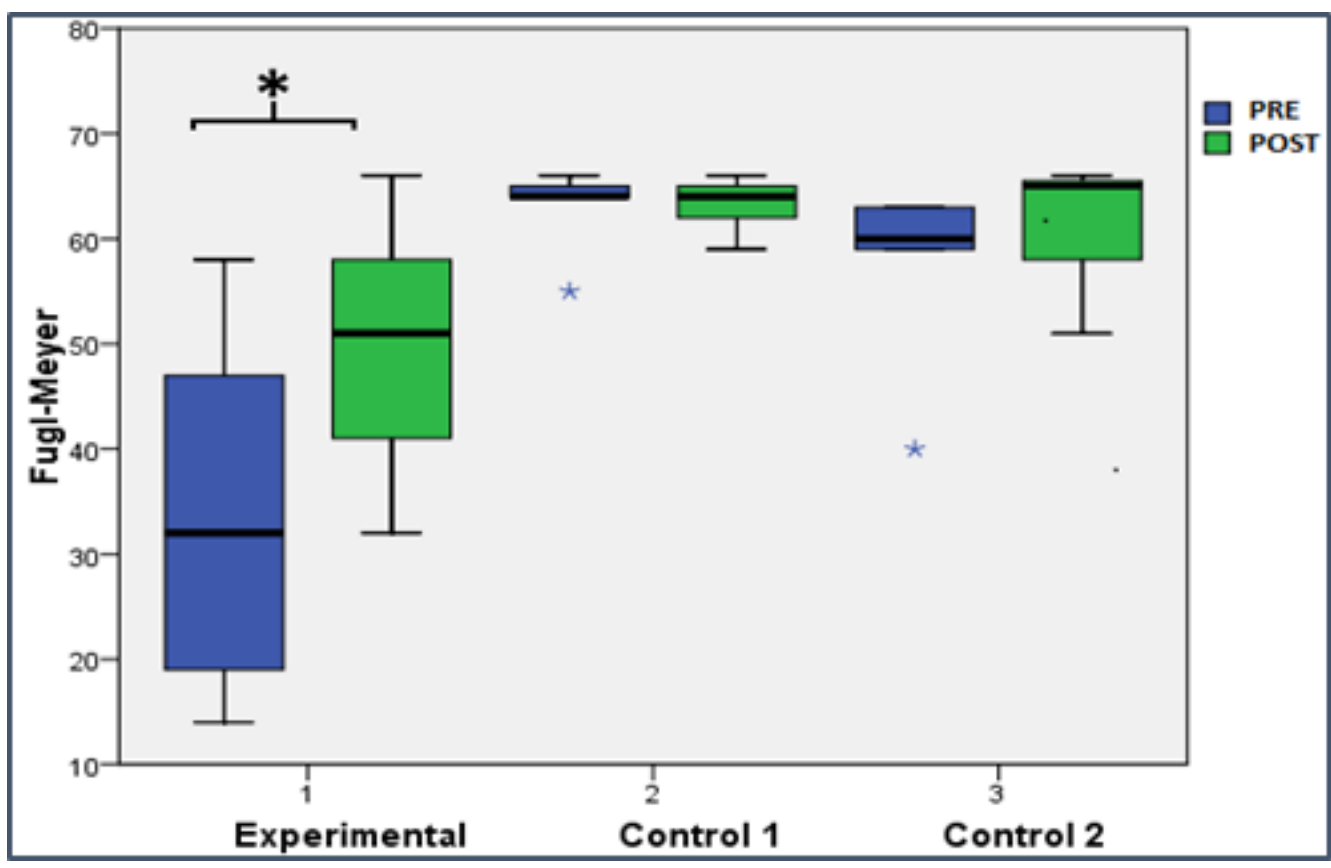

Figura 4. Comparación de los cambios en el test manual muscular para los extensores de muñeca de la extremidad superior parética en los diferentes grupo antes y después de la intervención.

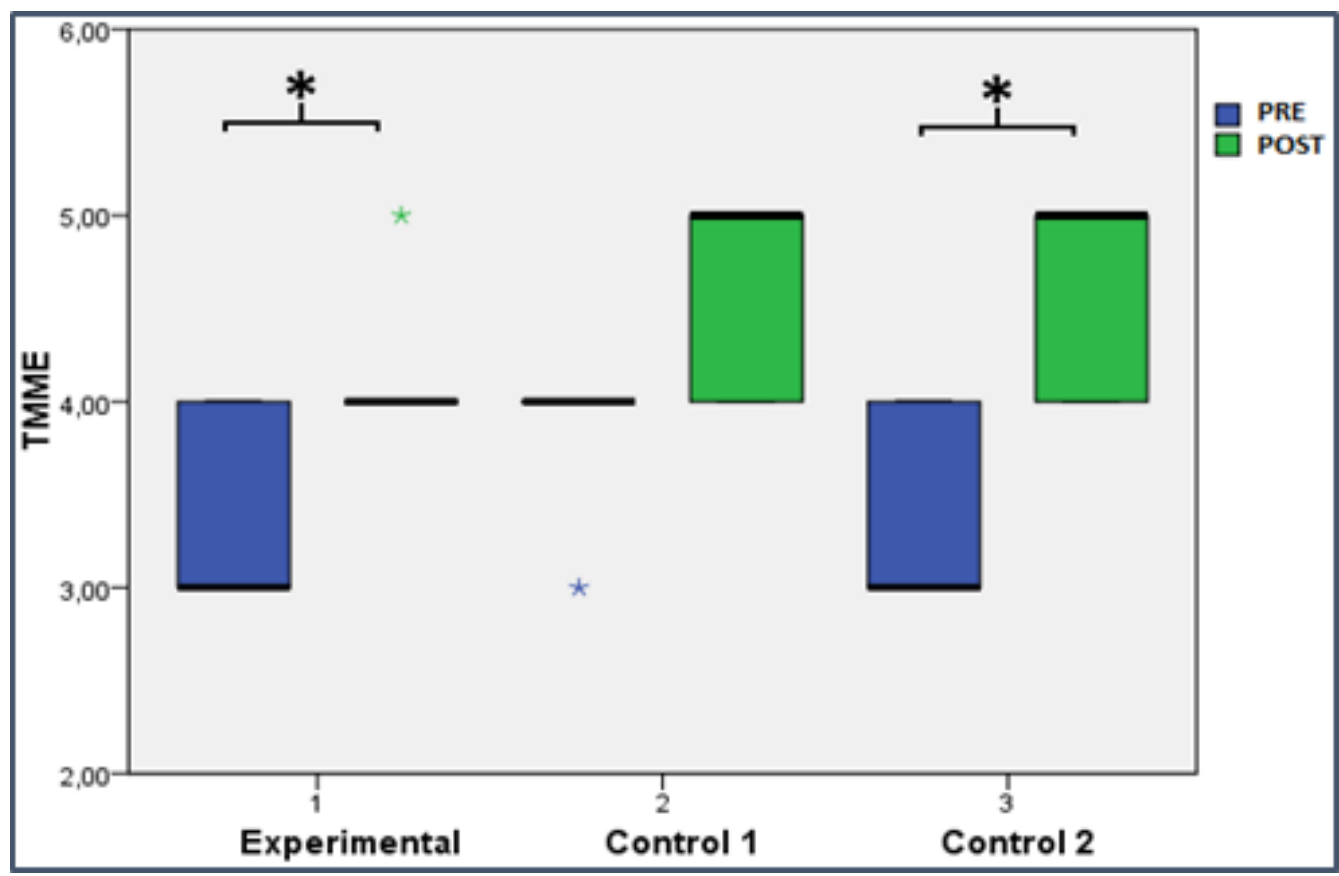


de los cambios en función de la extremidad superior se pueden correlacionar con cambios a nivel motor.

Es necesario incorporar nuevos elementos tecnológicos en rehabilitación, que favorezcan a la actualización de esta y sean asequibles, de bajo costo y que así los sujetos puedan entrenar mayor tiempo, junto con crear mejores protocolos de tratamiento y así potenciar la función de la mano por la importancia que tiene en las actividades cotidianas.

La no diferencia significativas de los dos grupos controles se atribuye a un efecto techo debido a que la mediana del valor inicial para todos los test fue muy elevada. Una forma de poder evitar esto con futuros estudios en utilizar además diferentes escalas como la escala de ARAT.

Aún falta el análisis de los datos en el seguimiento de los sujetos que se están pesquisando actualmente. Debido a esto, junto al bajo tamaño muestral, y la falta de mayor homogeneidad de la muestra, es que solo se puede sugerir que la aplicación de estimulación eléctrica funcional $y$ biofeedback en conjunto pueden ser un buen complemento a la terapia convencional pero falta mayor investigación en esta área.

\begin{abstract}
Resumen
Introducción El accidente cerebrovascular constituye la principal causa de muerte y discapacidad en Chile. Más del 85\% de los pacientes sufren hemiplejia y más del 69\% discapacidad motora funcional de las extremidades superiores. Esta alteración genera impacto en el uso de la extremidad superior, afectando las diversas actividades de la vida diaria de las personas. No existen estudios que combinen terapia de biofeedback electromiográfico y activación bimanual con estimulación eléctrica funcional en sujetos secuelados de Accidente cerebrovascular (ACV).Por esto, surge el interés de determinar el efecto que tiene un protocolo de entrenamiento basado en Estimulación Eléctrica Funcional (EEF) con activación bimanual y terapia de biofeedback en la función de la extremidad superior. Metodología: 15 sujetos secuelados de ACV entre 40 y 85 años reclutados en el área de neurorrehabilitación ambulatoria de Clínica Dávila, aleatorizados en un grupo experimental y en dos grupos controles de 5 sujetos. En cada sesión el grupo experimental entrenará quince minutos de activación bimanual con electroestimulación funcional y luego un programa de entrenamiento de biofeedback de diez minutos, mientras que el grupo control 1 y control 2 entrenaron en las mismas condiciones pero con EEF placebo y BF-EMG placebo respectivamente Resultados Existieron cambios significativos en el grupo experimental luego de la intervención. Conclusión Este estudio sugiere la estimulación eléctrica funciona y biofeedback como una herramienta para la rehabilitación de la extremidad superior paréticas en sujetos secuelados de ACV.

Palabras claves: Stroke, Neurofeedback, Rehabilitation
\end{abstract}




\section{Referencias Bibliográficas}

1. Guía Clínica AUGE. Accidente cerebrovascular isquémico en personas de 15 años y más. Serie Guías Clínicas MINSAL, 2013.

2. Ju-Hong Kim. The effects of training using EMG biofeedback on stroke patients upper extremity functions. J. Phys. Ther. Sci. 29: 1085-1088, 2017.

3. Hyun Seok, MD, PhD et al. Can ShortTerm Constraint-Induced Movement Therapy Combined With Visual Biofeedback Training Improve Hemiplegic Upper Limb Function of Subacute Stroke Patients?. Ann Rehabil Med 2016;40(6):998-1009 pISSN: 22340645 • eISSN: 2234-0653.

4. Kim HH, Kim KM, Chang MY: Intervenciones para promover la recuperación de la extremidad superior en pacientes con accidente cerebrovascular: una revisión sistemática . J Korean Soc Occup Ther , 2012, 129-145.

5. Pietrosimone, Brian, J. Troy Blackburn, Matthew S. Harkey, Brittney A. Luc, Derek N. Pamukoff, and Joe M. Hart. 2015. "Clinical Strategies for Addressing Muscle Weakness Following Knee Injury." Clinics in Sports Medicine 34 (2): 285-300.

6. Urra O, Casals A, Jane R.The impact of visual feedback on the motor control of the upper-limb. Conf Proc IEEE Eng Med Biol Soc. 2015;2015:3945-8.

7. Rehabilitation of Upper Extremity Nerve Injuries Using Surface EMG Biofeedback: Protocols for Clinical Application.

8. Nelson, Lonnie a. 2007. "The Role of Biofeedback in Stroke Rehabilitation: Past and Future Directions." Topics in Stroke Rehabilitation 14 (4): 59-66.

9. Giggins, Oonagh M, Ulrik Persson, Brian Caulfield, JJ Tate, CE Milner, J Onate, K Guskiewicz, et al. 2013.“Bio- feedback in Rehabilitation." Journal of NeuroEngineering and Rehabilitation 10 (1): 60.

10. Jonsdottir J, Cattaneo D, Regola A, Crippa A, Recalcati M, Rabuffetti M, Ferrarin M, Casiraghi A. Concepts of motor learning applied to a rehabilitation protocol using biofeedback to improve gait in a chronic stroke patient: an A-B system study with multiple gait analyses. Neurorehabil Neural Repair 2007;21:190-194.

11. Johanna Jonsdottir, ScD, PT, Davide Cattaneo, MA PT, Mauro Recalcati, PT, Alberto Regola, PT, Marco Rabuffetti, MA Eng, Maurizio Ferrarin, $\mathrm{PhD}$ Eng, and Anna Casiraghi, MD. Task-Oriented Biofeedback to Improve Gait in Individuals With Chronic Stroke: Motor Learning Approach. Neurorehabilitation and Neural Repair 24(5) 478-485.

12. Biofeedback improves performance in lower limb activities more than usual therapy in people following stroke: a systematic review.

13. Oonagh M Giggins, Ulrik McCarthy Persson and Brian Caulfield Biofeedback in rehabilitation.Journal of NeuroEngineering and Rehabilitation 2013.

14. Akkaya N1, Ardic F, Ozgen M, Akkaya S, Sahin F, Kilic A.Efficacy of electromyographic biofeedback and electrical stimulation following arthroscopic partial meniscectomy: a randomized controlled trial.Clin Rehabil. 2012 Mar;26(3):224-36.

15. Vanessa Draper. Electromyographic Biofeedback and Recovery of Quadriceps Femoris Muscle Function Following Anterior Cruciate Ligament Reconstruction. Physical Therapy, Volume 70, Issue 1, Pages 11-17. January 1990.

16. Nelson LA1. The Role of Biofeedback 
in Stroke Rehabilitation: Past and Future Directions. Top Stroke Rehabil. 2007 Jul-Aug;14(4):59-66.

17. Taub R1, Uswatte G, King DK, Morris D, Crago JE, Chatterjee A..A placebo-controlled trial of constraint-induced movement therapy for upper extremity after stroke Stroke 2006 Apr;37(4):1045-9. Epub 2006 Mar 2.

18. Recovery of supraspinal control of leg movement in a chronic complete flaccid paraplegic man after continuous low-frequency pelvic nerve stimulation and FES-assisted training.

19. Howlett O Using functional electrical stimulation with stroke survivors: A survey of Victorian occupational therapists and physiotherapists.Aust Occup Ther J. 2018 Aug;65(4):306-313.

20. Jonsdottir $\mathrm{J}$ et al. Arm rehabilitation in post stroke subjects: A randomized controlled trial on the efficacy of myoelectrically driven FES applied in a task-oriented approach. PLoS One. 2017 Dec 4;12(12):e0188642.

21. Cuesta-Gómez et al. The Use of Functional Electrical Stimulation on the Upper Limb and Interscapular Muscles of Patients with Stroke for the Improvement of Reaching Movements: A Feasibility Study.Front Neurol. 2017 May 10;8:186.

22. Nakipoğlu Yuzer GF et al. A Randomized Controlled Study: Effectiveness of Functional Electrical Stimulation on Wrist and Finger Flexor Spasticity in Hemiplegia. J Stroke Cerebrovasc Dis. 2017 Jul;26(7):1467-1471.

23. Eraifej $\mathrm{J}$ et al. Effectiveness of upper limb functional electrical stimulation after stroke for the improvement of activities of daily living and motor function: a systematic review and meta-analysis. Syst Rev. 2017 Feb 28;6(1):40.

24. Schuster-Amft C, Eng K, Suica Z, Thaler I,Signer S, Lehmann I, et al. (2018) Effect of a four-week virtual reality-based training versus conventional therapy on upper limb motor function after stroke: A multicenter parallel group randomized trial. PLoS ONE 13(10): e0204455.

25. Amano S1, Umeji A1, Uchita A1, Hashimoto Y1, Takebayashi T2, Kanata Y2, Uchiyama Y2, Domen K2.Reliability of remote evaluation for the $\mathrm{Fu}-$ gl-Meyer assessment and the action research arm test in hemiparetic patients after stroke. Top Stroke Rehabil. 2018 Sep;25(6):432-437.

26. Bernhardt Julie et al. Agreed definitions and a shared vision for new standards in stroke recovery research: The Stroke Recovery and Rehabilitation Roundtable taskforce International Journal of Stroke, 12(5) April 2017.
Correspondencia:

Sebastián Eduardo Alarcón Arancibia

Dirección postal: 7510891

alarcon88_8@hotmail.com

Teléfono del autor: +56981987583 